\title{
Differential expression of HMCES in glioblastoma.
}

Shahan Mamoor, MS1

1shahanmamoor@gmail.com

Glioblastoma multiforme is a brain cancer of the glial cells $(1,2)$. It is the most common brain cancer in adults and offers a 15 month median survival at diagnosis $(1,2)$. The poor outlook for patients diagnosed with glioblastoma demands an unbiased, systematic understanding of the transcriptional landscape of the glioblastoma tumor. We performed such an analysis by comparing the transcriptomes of glioblastoma tumors with that of the transcriptomes from control non-affected brain tissue samples using independently published microarray data $(3,4)$. We found that HMCES, or 5-hydroxymethylcytosine $(\mathrm{hmC})$ binding, ES cell-specific, a molecule involved in the protection of genomic integrity through shielding of abasic single-stranded DNA sites (5) was among the genes most differentially expressed by glioblastoma tumors when compared to brain tissue. This is the first report of differential expression of HMCES in glioblastoma tumors.

Keywords: glioblastoma multiforme, glioblastoma, systems oncology, HMCES, targeted cancer therapeutics, tumor transcriptome analysis.

1 OF 10 


\section{Introduction}

Glioblastoma is the most common brain cancer in adults and offers a 15 month median survival prognosis to those diagnosed $(1,2)$. Standard of care includes surgery if the tumor is operable, radiation therapy, and medical therapy including temozolomide and an implantable, nitrogen mustard chemotherapy-containing wafer called polifeprosan 20 with carmustine (5-8). The lack of targeted treatment options and dismal prognostic outlook demand efforts be directed toward a basic understanding of the transcriptional nature of the glioblastoma tumor as compared to the tissue in which it arises - the brain. We performed such an analysis here, comparing the microarray tumor transcriptome data from patients with glioblastoma multiforme to that of global transcriptional data from the normal brain $(3,4)$. We found that a molecule involved in the recognition of the methyl-cytosine mark in embryonic stem cells, HMCES, or 5hydroxymethylcytosine binding, ES-cell specific, was among the genes whose expression was most different between glioblastoma tumors and normal brain tissue. HMCES was recently reported to be sensor of abasic sites in single-stranded DNA, by cross-linking DNA and protein and these sites shielding it from error-prone DNA replication (5), and we found that HMCES was expressed at significantly lower levels in the tumors of patients with glioblastoma than in benign, non-affected brain tissue. This suggests that down-regulation of DNA repair at single-stranded DNA sites by HMCES may be an important event in the initiation, maintenance or progression of the glioblastoma tumor.

\section{Methods}

GSE116520 (3) and GSE108474 (4) were also used for this differential gene expression analysis, in conjunction with GEO2R. The transcriptional data from GSE116520, GBM tumor 2 OF 10 
from 17 patients diagnosed with glioblastoma multiform and normal brain from 8 controls, was generated by Kruthika et al. using Illumina HumanHT-12 V4.0 expression beadchip technology. The transcriptional data from GSE108474, GBM tumor from 221 patients diagnosed with glioblastoma multiform and normal brain tissue from 28 controls, was generated by Gusev et al. using Affymetrix Human Genome U133 Plus 2.0 Array technology. The $p$-value adjustment option was set to "Benjamini and Hochberg", application of log transformation to data was set to "Auto-detect" and the NCBI generated category of platform annotation was used. Statistical tests to compare the significance of RNA expression values between tumor and control groups was performed using a two-tailed, unpaired t-test with Welch's correction (PRISM 8.1.2) (227).

\section{Results}

We performed systems-level, comparative transcriptome profiling in glioblastoma by comparing the transcriptomes of 17 glioblastoma tumors from human patients to that of 8 nonaffected, benign brain tissue samples. We found that, when considering the entire all of the transcripts produced in the transcriptome, HMCES was among these genes whose expression was most significantly different glioblastoma tumors and healthy brain tissue.

HMCES is differentially expressed in the tumors of patients with glioblastoma when compared to non-affected benign brain tissue.

In the first dataset we analyzed, HMCES was the 168th most differentially expressed gene relative to the brain transcriptome in health, considering 47,229 mRNA transcripts in total. This was statistically significant (Table $1 ; p=3.39 \mathrm{E}-13$ ).

We probed a second dataset (4), tumor microarray data from the REMBRANDT study, a collection of genomic data from patients with brain cancers, to determine whether differential expression of HMCES could be observed in glioblastoma tumors from a separate group of patients. When sorting all of the genes expressed in 221 glioblastoma tumors based on change 3 OF 10 
in mRNA levels between GBM tumor and normal brain, HMCES ranked 24348 out of 54613 transcripts. Differential expression of HMCES in glioblastoma tumors was statistically significan (Table 2; $\mathrm{p}=3.41 \mathrm{E}-03$ ), albeit less so than we observed in the first dataset interrogated.

HMCES is expressed at significantly lower levels in glioblastoma tumors when compared to the brain.

Next, we obtained the exact mRNA expression values for the HMCES transcript in each of 17 glioblastoma tumors and in the 8 reference brain tissue samples. We found that HMCES was expressed at lower levels in the tumors of patients with glioblastoma as compared to nonaffected, control brain tissue. Decreased expression of HMCES in glioblastoma tumors was statistically significant (Figure $1 ; p<0.0001$ ).

\section{Discussion}

Glioblastoma is the most common brain tumor in adults, presents a 15 month median survival to those diagnosed with it and there are zero targeted treatments available for this disease. We performed global differential gene expression analysis of glioblastoma tumors compared to the brain in health using published microarray data (3) and integrated the results from that analysis with a separate interrogation of the REMBRANDT study to replicate results in a separate patient dataset (4). We found that HMCES, 5-hydroxymethylcytosine, ES-cell specific was among the genes whose expression was most different between the glioblastoma tumor and benign brain tissue in health. HMCES was expressed at significantly lower levels in the tumors of patients with glioblastoma as compared to the non-affected, control brain tissue in health (3).

HMCES was recently reported to function as a sensor of abasic sites in single-stranded DNA, by forming protein-DNA crosslinks, recruiting the proliferating cell nuclear antigen, PCNA, and by forming this cross-link, shielding these sites from error-prone DNA replication (5). Its 4 OF 10 
function protects these abasic, single-stranded DNA sites from translesion synthesis. By protecting single-stranded DNA molecules from replication using translesion DNA polymerases (TLS), HMCES prevents the formation of lesions, or mutations in DNA. Thus, HMCES was reported to be a protect integrity of the cellular genome by preventing formation of such translesion mutations at single-stranded sites.

Mutation is the driving force of evolution (9). We propose that by down-regulating HMCES, glioblastoma tumors promote the formation of mutations at single-stranded, a basic DNA sites and thus generate and select for the cellular environment most optimal for glioblastoma tumor progression. The function of HMCES in the glioblastoma, by genetic inhibition, and the effect of HMCES over-expression on glioblastoma proliferation in vitro, as well as in vivo using xenograft mouse models must be assessed $(10,11)$. Fifteen percent of glioblastoma tumors manifest hypermutation at relapse in the most highly expressed genes (12). Over-expression or activation of HMCES function presents an novel and targeted therapeutic strategy to reduce the ability of the glioblastoma tumor to evade existing treatment options by increasing rate of mutation and producing mutant clones that can resist chemotherapy and radiation treatment.

\section{References}

5 OF 10 
1. Davis, M.E., 2016. Glioblastoma: overview of disease and treatment. Clinical journal of oncology nursing, 20(5), p.S2.

2. Alexander, B.M. and Cloughesy, T.F., 2017. Adult glioblastoma. Journal of Clinical Oncology, 35(21), pp.2402-2409.

3. Kruthika, B.S., Jain, R., Arivazhagan, A., Bharath, R.D., Yasha, T.C., Kondaiah, P. and Santosh, V., 2019. Transcriptome profiling reveals PDZ binding kinase as a novel biomarker in peritumoral brain zone of glioblastoma. Journal of neuro-oncology, 141(2), pp.315-325.

4. Gusev, Y., Bhuvaneshwar, K., Song, L., Zenklusen, J.C., Fine, H. and Madhavan, S., 2018. The REMBRANDT study, a large collection of genomic data from brain cancer patients. Scientific data, 5, p.180158.

5. Mohni, K.N., Wessel, S.R., Zhao, R., Wojciechowski, A.C., Luzwick, J.W., Layden, H., Eichman, B.F., Thompson, P.S., Mehta, K.P. and Cortez, D., 2019. HMCES maintains genome integrity by shielding abasic sites in single-strand DNA. Cell, 176(1-2), pp.144-153.

6. Stupp, R., Mason, W.P., Van Den Bent, M.J., Weller, M., Fisher, B., Taphoorn, M.J., Belanger, K., Brandes, A.A., Marosi, C., Bogdahn, U. and Curschmann, J., 2005. Radiotherapy plus concomitant and adjuvant temozolomide for glioblastoma. New England Journal of Medicine, 352(10), pp.987-996.

7. McGirt, M.J., Than, K.D., Weingart, J.D., Chaichana, K.L., Attenello, F.J., Olivi, A., Laterra, J., Kleinberg, L.R., Grossman, S.A., Brem, H. and Quiñones-Hinojosa, A., 2009. Gliadel (BCNU) wafer plus concomitant temozolomide therapy after primary resection of glioblastoma multiforme. Journal of neurosurgery, 110(3), pp.583-588.

8. Affronti, Mary Lou, Christopher R. Heery, James E. Herndon, Jeremy N. Rich, David A. Reardon, Annick Desjardins, James J. Vredenburgh, Allan H. Friedman, Darell D. Bigner, and Henry S. Friedman. "Overall survival of newly diagnosed glioblastoma patients receiving carmustine wafers followed by radiation and concurrent temozolomide plus rotational multiagent chemotherapy." Cancer: Interdisciplinary International Journal of the American Cancer Society 115, no. 15 (2009): 3501-3511.

9. Wright, S., 1932. The roles of mutation, inbreeding, crossbreeding, and selection in evolution (Vol. 1, pp. 356-366). na.

10. Saito, R., Bringas, J.R., Panner, A., Tamas, M., Pieper, R.O., Berger, M.S. and Bankiewicz, K.S., 2004. Convection-enhanced delivery of tumor necrosis factor-related apoptosisinducing ligand with systemic administration of temozolomide prolongs survival in an intracranial glioblastoma xenograft model. Cancer research, 64(19), pp.6858-6862.

11. Giannini, C., Sarkaria, J.N., Saito, A., Uhm, J.H., Galanis, E., Carlson, B.L., Schroeder, M.A. and James, C.D., 2005. Patient tumor EGFR and PDGFRA gene amplifications retained in an invasive intracranial xenograft model of glioblastoma multiforme. Neurooncology, 7(2), pp.164-176. 
12. Wang, J., Cazzato, E., Ladewig, E., Frattini, V., Rosenbloom, D.I., Zairis, S., Abate, F., Liu, Z., Elliott, O., Shin, Y.J. and Lee, J.K., 2016. Clonal evolution of glioblastoma under therapy. Nature genetics, 48(7), p.768. 
HMCES

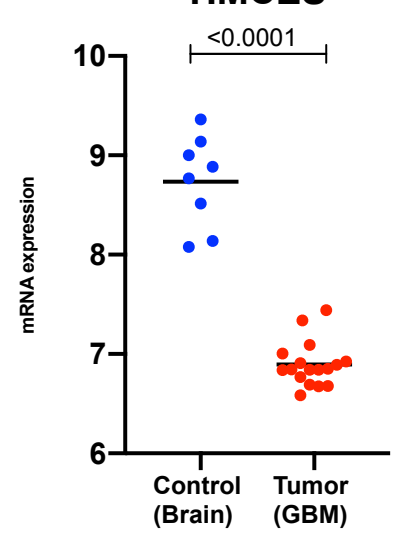

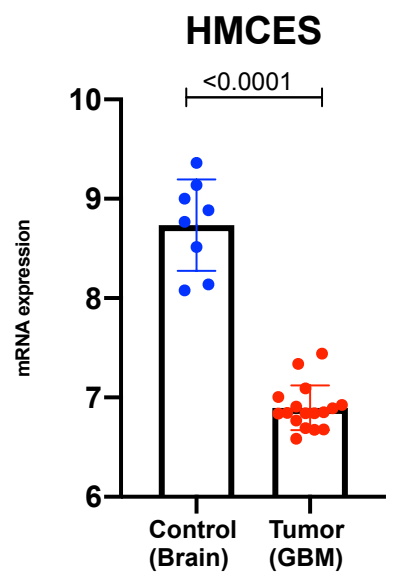

HMCES

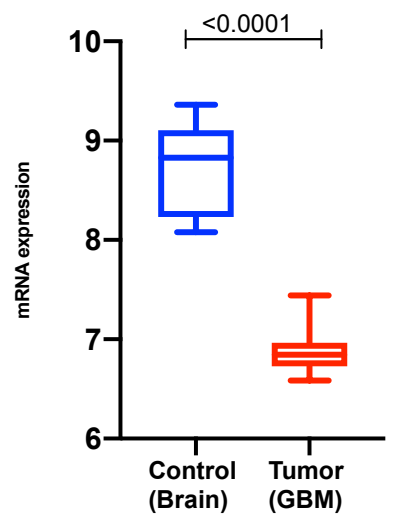

Figure 1: HMCES is expressed at significantly lower levels in glioblastoma tumors when compared to the brain.

The mRNA expression level of HMCES is graphically represented by dots denoting the expression level in each individual patient and the horizontal black bar representing the mean expression value $(A)$; as a bar graph with the error bars denoting the standard error of the mean (B); and as box and whisker plots from minimum to maximum value (C). 
Table 1: HMCES is differentially expressed in the tumors of patients with glioblastoma when compared to non-affected benign brain tissue.

The rank of HMCES differential expression, $p$-value with respect to global differential expression, t-statistic, B, the log-odds of differential expression between the two groups (tumor and control), gene symbol, gene name, as well as Illumina probe ID (all provided by GEO2R) are shown here. 


\begin{tabular}{l|l|l|l|l|l|l|}
\hline Rank & ID & p-value & t & B & Gene & Gene name \\
\hline 24348 & 201678_s_at & 3.41 E-03 & 2.95592 & -2.81054 & HMCES & $\begin{array}{l}\text { 5-hydroxymethylcytosine } \\
\text { (hmC) binding, ES cell- } \\
\text { specific }\end{array}$ \\
\hline
\end{tabular}

Table 2: Statistically significant differential expression of HMCES in glioblastoma tumors from the REMBRANDT study.

The rank of HMCES differential expression, probe/transcript ID, $p$-value with respect to global differential expression, t-statistic, $\mathrm{B}$, the log-odds of differential expression between the two groups (tumor and control), gene symbol and gene name are shown here. 